\title{
GROWTH IDENTIFICATION OF TOMATO PLANTS USING TENSOR FLOW
}

\author{
Bharath Kumar G, Hemanth V, Balaji K.P, Makinani Gowri Shankar M, Mrs Tupili Sangeetha \\ JNN Institute Of Engineering, \\ Chennai, Tamil Nadu
}

\begin{abstract}
Vertical Farming is making the necessary daily vegetables available in a minimal space with effective growth. Tomato plant farming is one of it. In tomato plant farming growth monitoring is a key issue which require man-work and experience. In this project work, an attempt has been made to Analyse and Design for a growth identification of tomato plants. This project work involves planning, analysis, designs, and many more to identify growth stages. Vertical Farming is making the necessary daily vegetables available in a minimal space with effective growth. Tomato plant farming is one of it. In tomato plant farming growth monitoring is a key issue which require man-work and experience. To make it effective we are intruding Neural Networks for growth identification for Tomato plant which not require man-work. Without the involvement of man work, we track the plant growth with Neural Networks. And also, we are doing plant stage detection it shows the entire information of that plant such as temperature, quantity of water needed, etc. With the help of an IOT device all the data are stored in a database. We use raspberry pie IOT device and image sensor to store all the data to the database created.
\end{abstract}

Keywords - Plant Stage Detection, Vertical Farming, Image Sensor, Four Level Of Growth Stages

\section{INTRODUCTION}

Vertical farming is the modern practice of producing food that comes in different shapes and sizes either horizontally as shelving units or vertically as staircases. This soilless farming developing plants in controlled indoor atmosphere, with artificial lightening that provides adequate room temperature for photosynthesis process, water flow with nutrients mix (NPK ratios), and $\mathrm{CO} 2$ etc. Vertical farming uses 70-95 less water compared to traditional way of farming. And crops in this farming are free from unfavourable weather conditions and assures more crop productions which is pesticide free and pure organic. Till now, many believe that vertical farming can be the answer that can fulfil these food demands to certain extent.
In this paper, presentation of an "Growth identification of tomato plants" built for the purpose for vertical farming. Tomato plant require nutrients, water and co2 with variation in quantity at each stage to make growth more effective. This will help plant to grow more stronger and healthier. In tomato plant there are several stages while growing. Each growing stage takes some time to grow to next level. For example, in tomato plant seeding stage is the first stage in tomato plant. Growing level from seeding stage to early stage it takes up to $10-15$ days. For every tomato plant nutrients and water is important for growing if we put less amount of nutrients and water for a plant it will not grow healthy. Many plants are dyeing now a days because of lack of nutrients and water supply. In these days. Man, only handled the process in day to day life and it has been successful till the date. Our project idea does not require man power to handle for growing. With the help of the images we will identify which amount of nutrients to be added for each stage. This makes the job much easier and it will help to grow many plants in day to day life. In our project we are calculating the first four stages of tomato plant the seeding, early, vegetation and flowering. In these stages we calculate what amount of nutrients to be added and deliver that message to the customers. The information helps the customer/farmers to grow their plant much healthy and faster. We have currently done this for the first four stages of growing that will be very in vertical farming. Finally, plant stage detection is made.

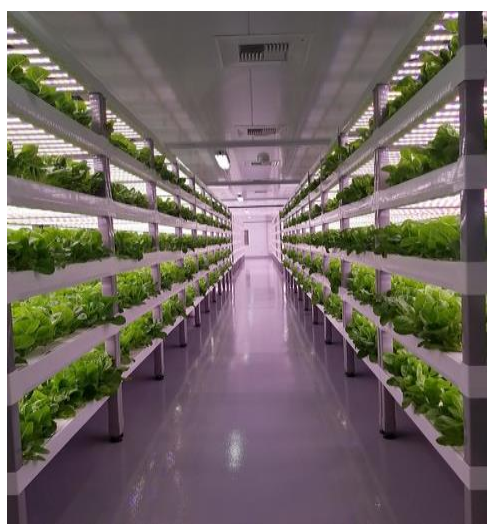

Figure 1.1 - Vertical Farming 


\section{RELATED WORK}

This study focuses on the feature extraction and development of tomato growth model. In Indonesia, the application of sensors and actuators to build smart farming has become increasingly popular in the past year but to produce measured harvest quality and quantity in smart farming, a model of growth and yield of the tomato plant is needed. in this paper, observations on the growth of tomato plants were carried out to extract features in the growth of tomato plants. In the experiment, a greenhouse was built to maintain the growth conditions of tomato plants which are equipped with an information system for reading sensor data to determine the greenhouse environment conditions for the growth of tomato plants with a local-global management strategy. From the experiment, we obtained a response model of the tomato plant growth with the effect of the maximum temperature that caused the blossoms to fall. and from the growth model, there are 5 growth stages of tomato plants are obtained, which at each stage has different growth variables with different nutritional needs and environmental conditions. with the important knowledge gained from this experiment, it can be used to develop a dynamic tomato plant growth model based on the growth stage, which is expected to be applied in the control system on smart farming in the future work.

In Indonesian agriculture, horticulture is one of the important sectors. Centre for Agricultural Data and Information System Ministry of Agriculture Republic of Indonesia (2016) [1] reported there are 2.9 million farmers in the horticulture sector. This sector mainly produces fruits, vegetables, herbal crop, and ornamental plants which is an opportunity to increase farmers' income and welfare, as well as improve people nutrition status [2]. So as to improve production systems from agricultural environment factors must be considered. many approaches have been proposed by researchers to optimize agricultural processes such as the WSN application to monitor environmental conditions [3]- [5]. Development of a remote environmental monitoring and control framework for tropical horticulture [6] and webbased monitoring [7]. Many researchers also study in the controlled environmental agricultural system using greenhouse. Crop growth model generally developed to help farmers manage the agricultural process. The objective of this experiment was (a) feature extraction of tomato plant growth on environmental conditions. (b) observe the stages of tomato plants growth, how to measure growth at each growth stage, the need for nutrition and environmental conditions at each growth stage. These are the features we added compared to the existing projects. In recent times, detection of unhealthy region of plant leaves using image processing and genetic algorithm process is done by some authors [8]. It detects the plant leaves and it sends the information. In this idea a detecting the plant is taken to our project to identify the growth stages of tomato plants using tensor flow. In 2017, A modified web-based agro-climatic remote monitoring system via wireless sensor network work is done [9]. It helps to identify the agro remote monitoring using wireless sensor network. In this idea wireless sensor concept is taken for our project to capture the images tomato plant growth stages. In 2016, development of a remote environmental monitoring and control framework for tropical horticulture and verification of its validity under unstable network connection in rural area is done [10]. In this idea they developed environment monitoring system for the purpose of rural area. For our project follows the same monitoring system of each plant growth stages using the server. In 1991, the logistic function-its application to the description and prognosis of plant growth project has been implemented [11]. For preparing nutrients charts to our project for the purpose of tomato plant growth stages, this project helps us to implement this data chart to the server. Basically, vertical farming is the practice of growing crops in vertical slaked areas. In our project vertical farming helps for growing tomato plants using the system software. And also, with the help of an IoT device called raspberry pie and image sensor also it helps to take images of the growing plants from stage by stage and send all the images to the database.

\section{PROPOSED SYSTEM}

We have proposed many features compared to the existing systems. since we are using IOT image sensor to collect images and process them to get growth determination results, it saves man work and time. For this, we train images with neuralnetworks which will recognize the coming images and identify the growth. After finding the each and every growth stages it sends the data to the database. In database it will compare the data with the existing data. By comparison, a message is sent to the customer or farmer which has some suggestions to make the growth more progressive. For this we use an IOT device called raspberry pie. This Raspberry Pi is a tiny and affordable computer that you can use to learn programming through fun, practical projects. For capturing the images, we use with neural networks. For this and image sensor helps to capture the images of each and every growth stages.

In our proposed, since we are using IOT image sensor to collect images and process them to get growth determination results, it saves man work 
and time. We train the images with neural-networks which will recognize the coming images and identify the growth. After finding the growth state, the data is sent to database which will compare with existing data. By comparison, a message is sent to the customer/ farmer which has some suggestions to make the growth more progressive. In this system it reduces the working time and it makes the plant to grow faster. Raspberry pie helps us to connect to the database for storing the data. We use image sensor to capture images. An image sensor or imager is a sensor that detects and conveys information used to make an image. We use front end language as python language. This language is used as a software requirement and it is main software that runs in the front end. In the back end of our project we run the database which stores each and every information. For our project any operating system is suitable. Operating systems helps to operate the functions like error detection and handling, handling I/O operations, etc. This whole system reduces the time for the customers/farmers for growing the tomato plants faster and healthiest. In the output of our project it detects the image and it delivers all the information that is required. And also, it will give them some suggestions if their plants are not grown effectively. This will help them a lot to grow their plant faster and healthier by the nutrients and water given regularly.

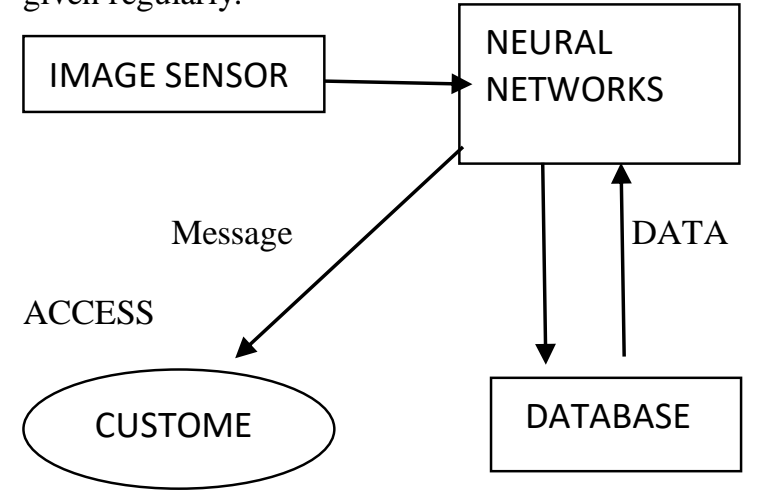

Figure 3.1 - Flow Diagram

\section{Stages Of Plant}

\section{Seeding Stage}

The plant life cycle starts from germination of seeds with available water, oxygen, light and right temperature at $75-89$ F. Seeding stage is the initial stage for growing a tomato plant. This is our first stage of our project. It takes upto10-15 days to go to next level of growing. The nutrients and water supply differs from each stage. For example, seeding stage requires 40: 24: $24 \mathrm{~kg} \mathrm{~N}$ : P: K/acre-for varieties and 60: 36: $36 \mathrm{~kg} \mathrm{~N}$ : P: K/acre-for hybrids for its first level of growing. Seeding stage is the initial stage for growing a tomato plant. This is our first stage of our project. It takes upto10-15 days to go to next level of growing. For every stage nutrients and water supply is needed.

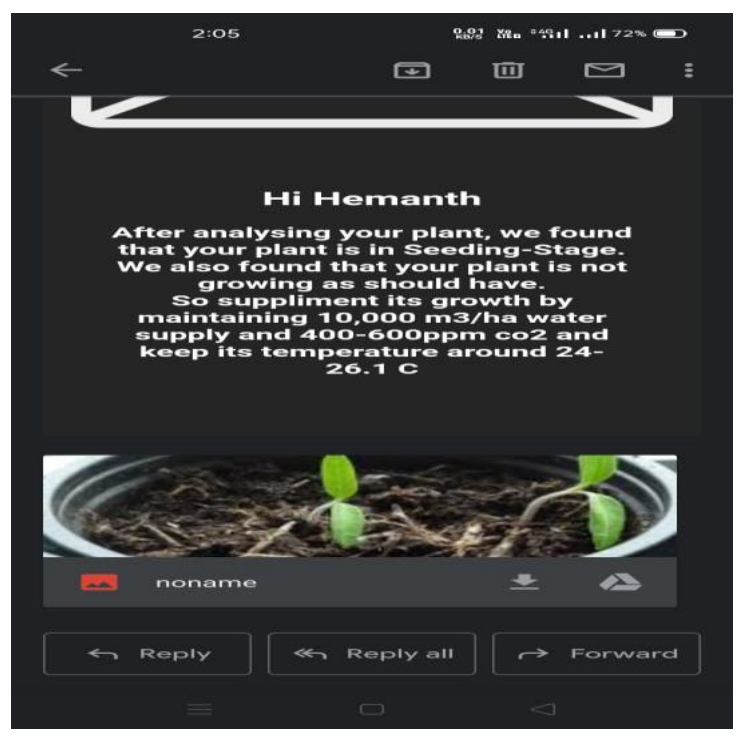

Figure 3.2 - Seeding Stage Plant Detection

\section{Early Stage}

This stage begins when plants are planted on the first day. Plants have begun to appear on the surface as illustrated in stage 1 in the 3rd day. This stage 1 stops on day 6 when the plants have raised 2 leaves and start to initiate more leaves and stems.

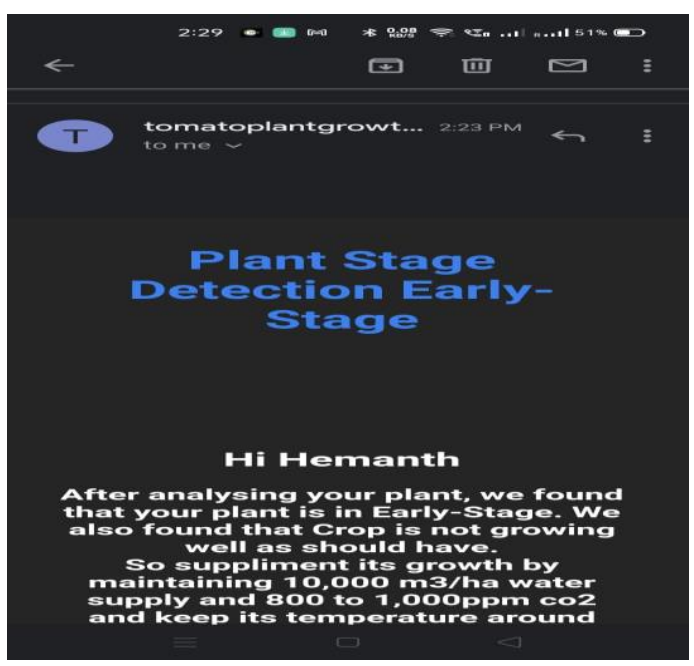

Figure 3.3 - Early Stage Detection

\section{Vegetation Stage}

In stage 3, leaves and stems will continue to initiate and grow exponentially until the first blossom appears. The appearance of blossoms occurs on the 50 th day based on the experiment. At this stage, the 
plant starts preparing its own food called as Photosynthesis. This period is actually green growing period and need more nutrients. It requires large amount of nitrogen for the growth of root, stem, branch and leaf areas. This is the third level of tomato plants while growing. This stage also requires some higher amount of nutrients and water supply compared to the first two stages. It grows faster compared to the older stages because it has more nutrients and water supply than the first two growing stages. It takes times of growing up to 1520 days from the start of growing.

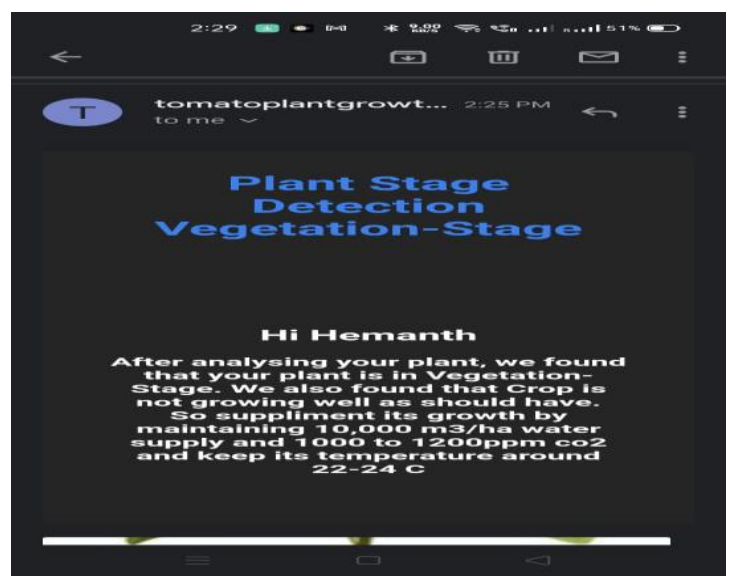

Figure 3.3- Vegetation Stage Detection

\section{Flowering Stage}

After going through the stage 3 , the stage 4 is where the leaves and stems growth rate decrease and blossoms will continue to appear until the first fruit formation, the fruit formation is the evidence of the end of stage 3. The first fruit formation appears on day 90 based on the experiment.

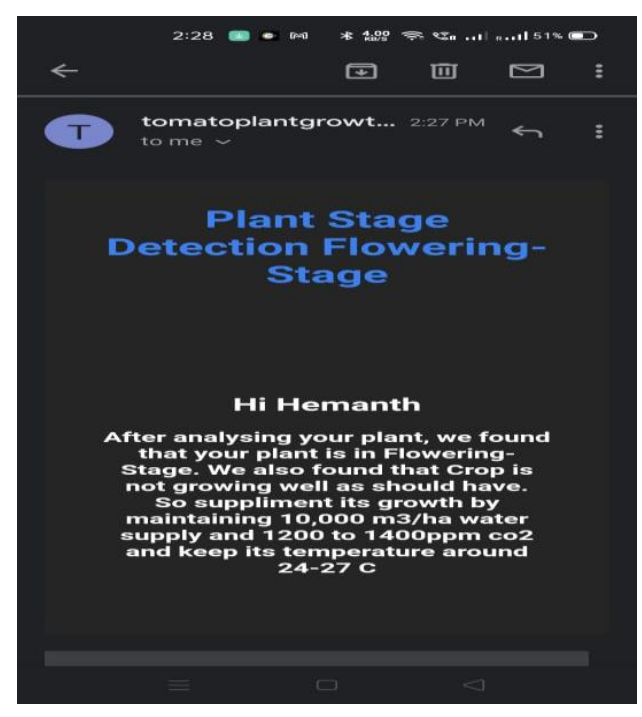

Figure 3.5 - Flowering Stage Detection

\section{Technology Used:}

Raspberry Pie - The Raspberry Pi is a tiny and affordable computer that you can use to learn programming through fun, practical projects. In our project it is our hardware requirements and it stores the each and every data in the database.

Image Sensor - An image sensor or imager is a sensor that detects and conveys information used to make an image.

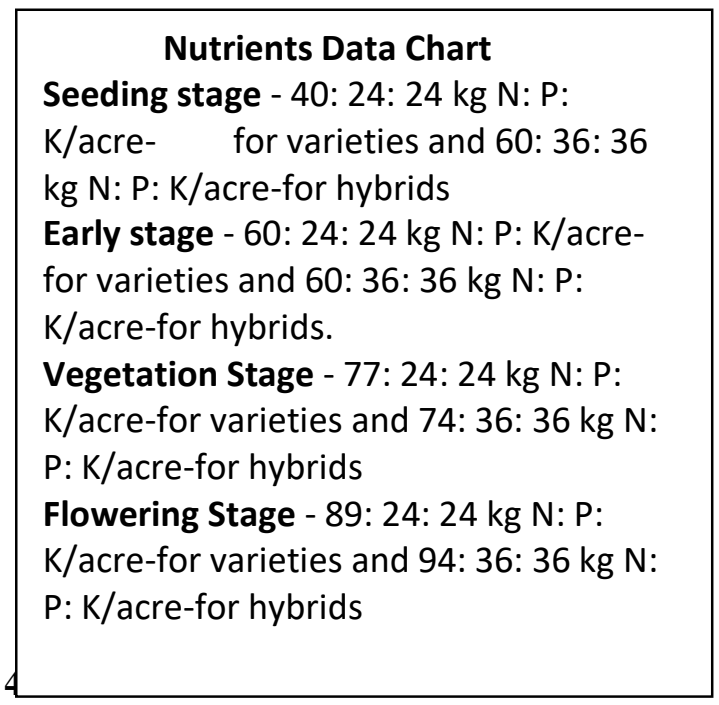

In this section the step by step process is explained briefly one by one for identify the growth stages of tomato plants. Each and every process is mentioned below: Main idea is to find the first four growth stages of tomato plants they are early, seeding, vegetation and flowering. For that, first we train all the images of the growth stages and process them to get growth determination results, it saves man work and time. We use IOT image sensor to capture images. We train all the images with neural networks this helps to recognize the coming images and identify the growth. After finding the growth state of each stages, the data is sent to database which will compare with existing data that will be already present. After the comparison, a message is sent to the customer/ farmer which has some suggestions to make the growth more progressive. With the information given to the customers. The customer/farmer can grow the plants very quickly and healthy.

\section{MODULE DESCRIPTION}

Collecting Data-Set - The data-set for plant stages are collected in various forms of online resources. These data-set contain four stages. The stages are used for growth identification of tomato plant. The stages are seeding stage, Early Stage, Vegetation and Flowering stage. 
Building Neural-Network - For the identification of images using image processing, we are using neural networks. The neural-network is trained by using tensor-flow libraries to make it easier to image classification. For the whole project we are using libraries like matplotlib, cv2, keras, numpy. To train the model we are using 3 layers of convulsion neural networks. It increases both robustness and accuracy.

Testing Model -After the model is trained with the existing data-set we will test the model accuracy and robustness by supplying unclassified image to the model and analyse the result. If the model is giving accurate result, we will implement it. if the model is not up-to the mark, then we will retrain the model and do the process again till we get the expected results.

Connecting Raspberry $\mathrm{Pi}$ - After the model is successfully predicting the images, now we will deploy this model into IOT device. In our case it is Raspberry Pi attached with camera. Now the trained model will run in the device by collecting the images using camera and gives the prediction.

Connection to Data-Base - After successfully deploying the model, now we will connect it to the data base which contains the nutritional chart for the tomato plant. By comparing the plant stage and the no of days it took to reach the stage we will give some suggest the farmer for more yield.

\section{SYSTEM ARCHITECTURE}

The system architecture is the key role for every projects. In our project, raspberry pie is used as a database where all the four stages of data are stored. First it captures images with the help of the image Sensor, it will capture images of the plant and first it sends the image to the raspberry and after then it Sends the image to the database for the purpose of comparison. After comparing with the existing data, it sends all the information required to the farmer like how much quantity amount of nutrients and water should be added for the next stage. All this information will be sent to the Emil-id of a farmer

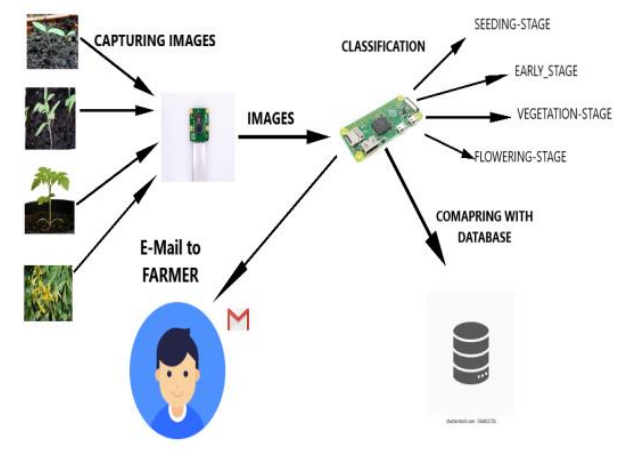

Figure 6.1- System Architecture Of Collecting Images

\section{CONCLUSION AND FUTURE WORK}

In this project, four growth stages are identified. The four growth stages of tomato plant are calculated with the help of the image processing and neural networks. And also, nutrients chart is created to calculate which amount of nutrients is taken for each and every stage. For this we have trained the model for each and every image to identify the stages. IOT image sensor is used to capture images. Python code is generated with raspberry pie that will send the data to the database. That data will compare with the existing data. At last it sends the information to the customers. With the help of the information the customer/farmer can grow their plants faster and healthy. Finally, in the output the plant stage detection will happen it tells the exact information of the plant and also it guides some information to go to next level of growing.

In future we are going to the find the height measurement scheme for tomato plant growth stages using Neural Networks.

\section{REFERENCES}

[1] S.s.Kumar., Farid Taher and Rahim Islam.(2006)'Artificial Intelligience And Image Processing Approaches In Damage Assessment And Material Evaluation. DOI: 10.1109/CIMCA.2005.1631284.

[2] Luthfi Aminulloh., Wahjoe TjaturSesulihatien., D. Pramadihanto.(2019)'

Feature Extraction Of Tomato Growth Model Using Greenhouse Monitoring System. DOI: 10.1109/ELECSYM.2019.8901534

[3] Prof. Bhavana Patil1., Mr.Hemant Panchal2., Mr. Shubham Yadav3., Mr. Arvind Singh4., Mr. Dinesh Patil5.(2017)' Plant monitoring using image processing, raspberry pie and IOT.

[4] Gupta N.K.1., Isaac R.K.2 and R.K. Singh3. (2016)' Maintenance and analysis of agricultural data: a challenge. DOI: 10.21746/ijbio.2016.09.0010

[5] J.-A. Jiang et al., (2018)' Toward a higher yield: a wireless sensor network-based temperature monitoring and fan-circulating system for precision cultivation in plant factories

[6] J.-A. Jiang et al., (2016)' A wireless sensor network-based monitoring system with dynamic 
converge cast tree algorithm for precision cultivation management in orchid greenhouses.

[7] Sachin. D.Khirade., A.B.patil.,(2015)' Plant disease detection Using image processing . International conference on computing communication control and automation, IEEE. J. Clerk Maxwell, A Treatise on Electricity and Magnetism, 3rd ed., vol. 2. Oxford: Clarendon, 1892, pp.68-73.

[8] Vijai singh, Varsha., A.K.Mishra.,(2010)' Detection of unhealthy region of plant leaves using image processing and genetic algorithm. 205, ICACEA, India. K. Elissa, "Title of paper if known," unpublished.

[9] J. Agajo.,J. G. Kolo.,G. Jonas., A. R. Opeyemi, N. O. Chikeze., O. B. Chukwujekwu.,(2017)' A modified web-based agro-climatic remote monitoring system via wireless sensor network. IEEE 3rd International Conference on ElectroTechnology for National Development (NIGERCON), 2017, pp. 258-270.

[10] A. P. Nugroho., (2016)' Development of a remote environmental monitoring and control framework for tropical horticulture and verification of its validity under unstable network connection in rural area. Comput. Electron. Agric., p. 15, 2016.

[11] A. Gregorczyk., (1991)' The logistic functionits application to the description and prognosis of plant growth." Acta Soc. Bot. Pol., vol. 60, no. 1-2, pp. 67-76, 1991. 